\title{
COMPOSITION OF ARITHMETICAL IDENTITIES
}

BY E. T. BELL

1. Examples of Composition. This concerns a new type of arithmetical identity involving parity functions. The letters $n$, $d, \delta, m, t, \tau$, with or without accents or suffixes, denote integers greater than zero; the $n, d, \delta$ are arbitrary, the $m, \tau$, odd. Hence, if $n=t \tau, t$ is a divisor of $n$ whose conjugate divisor $(\tau)$ is odd. In what immediately follows, $m, n$ (without suffixes or accents) are constant; summations refer to all values of the variable integers in the indicated partitions consistent with the notation just explained.

Let $f(x, y)$ be single-valued and finite for all pairs of integer values of $x, y$, and let $f(x, y)$ be even in each of $x, y$,

$$
f(x, y)=f(-x, y)=f(x,-y) .
$$

Then there are the following known arithmetical identities, proofs of which will be indicated in $\$ 2$.

(I) $\quad 2 n=m_{1}+m_{2} ; \quad n=t \tau, \quad m_{1}=t_{1} \tau_{1}, \quad m_{2}=t_{2} \tau_{2}$ :

$\sum\left[f\left(t_{1}+t_{2}, \tau_{1}-\tau_{2}\right)-f\left(t_{1}-t_{2}, \tau_{1}+\tau_{2}\right)\right]=\sum t[f(2 t, 0)-f(0,2 t)]$.

(II) $\quad n=n_{1}+n_{2} ; \quad n=d \delta, \quad n_{1}=d_{1} \delta_{1}, \quad n_{2}=d_{2} \delta_{2}$ :

$\sum\left[f\left(d_{1}+d_{2}, \delta_{1}-\delta_{2}\right)-f\left(d_{1}-d_{2}, \delta_{1}+\delta_{2}\right)\right]$

$$
=\sum\left[(d-1)\{f(d, 0)-f(0, d)\}+2 \sum_{r=1}^{\delta-1}\{f(d, r)-f(r, d)\}\right] .
$$

A sum in which the upper limit exceeds the lower is vacuous, by convention.

Repeating (I) in accented letters, we have ( $n^{\prime}$ is constant)

$$
\begin{gathered}
2 n^{\prime}=m_{1}^{\prime}+m_{2}^{\prime} ; \quad n^{\prime}=t^{\prime} \tau^{\prime}, \quad m_{1}^{\prime}=t^{\prime} \tau_{1}^{\prime}, \quad m_{2}^{\prime}=t_{2}^{\prime} \tau_{2}^{\prime}: \\
\sum\left[f\left(t_{1}^{\prime}+t_{2}^{\prime}, \tau_{1}^{\prime}-\tau_{2}^{\prime}\right)-f\left(t_{1}^{\prime}-t_{2}^{\prime}, \tau_{1}^{\prime}+\tau_{2}^{\prime}\right)\right] \\
=\sum t^{\prime}\left[f\left(2 t^{\prime}, 0\right)-f\left(0,2 t^{\prime}\right)\right] .
\end{gathered}
$$

From (I) and $\left(\mathrm{I}^{\prime}\right)$, and hence from (I) alone, we can infer by elementary arithmetical manipulations the following identity, which we number $\left(\mathrm{I}, \mathrm{I}^{\prime}\right)$ for a reason appearing in $\$ 2 ; n, n^{\prime}$ are 
constant; $\sigma_{1}\left(n^{\prime}\right) \equiv \zeta_{1}\left(n^{\prime}\right)+\zeta_{1}^{\prime}\left(n^{\prime}\right), \zeta_{1}\left(n^{\prime}\right) \equiv$ the sum of the divisors of $n^{\prime}, \zeta_{1}^{\prime}\left(n^{\prime}\right) \equiv$ the sum of the odd divisors of $n^{\prime}$.

$$
\begin{aligned}
& \text { (I, I') } \quad 2 n=m_{1}+m_{2} ; \quad n=t \tau, \quad m_{1}=t_{1} \tau_{1}, \quad m_{2}=t_{2} \tau_{2} \text {; } \\
& 2 n^{\prime}=m_{1}^{\prime}+m_{2}^{\prime} ; \quad n^{\prime}=t^{\prime} \tau^{\prime}, \quad m_{1}^{\prime}=t_{1}^{\prime} \tau_{1}^{\prime}, \quad m_{2}^{\prime}=t_{2}^{\prime} \tau_{2}^{\prime}: \\
& \sum\left[f\left(\left(t_{1}+t_{2}\right)\left(t_{1}^{\prime}-t_{2}^{\prime}\right),\left(\tau_{1}-\tau_{2}\right)\left(\tau_{1}^{\prime}+\tau_{2}^{\prime}\right)\right)\right. \\
& -f\left(\left(t_{1}+t_{2}\right)\left(t_{1}^{\prime}+t_{2}^{\prime}\right),\left(\tau_{1}-\tau_{2}\right)\left(\tau_{1}^{\prime}-\tau_{2}^{\prime}\right)\right) \\
& -f\left(\left(t_{1}-t_{2}\right)\left(t_{1}^{\prime}-t_{2}^{\prime}\right),\left(\tau_{1}+\tau_{2}\right)\left(\tau_{1}^{\prime}+\tau_{2}^{\prime}\right)\right) \\
& \left.+f\left(\left(t_{1}-t_{2}\right)\left(t_{1}^{\prime}+t_{2}^{\prime}\right),\left(\tau_{1}+\tau_{2}\right)\left(\tau_{1}^{\prime}-\tau_{2}^{\prime}\right)\right)\right] \\
& =\sum t\left[\sigma_{1}\left(n^{\prime}\right) f(0,0)-t^{\prime}\left\{f\left(4 t t^{\prime}, 0\right)+f\left(0,4 t t^{\prime}\right)\right\}\right] .
\end{aligned}
$$

In the same way (II), (I) give the next, in which $n, n^{\prime}$ are constant.

$$
\begin{aligned}
& \left(\mathrm{II}, \mathrm{I}^{\prime}\right) \quad n=n_{1}+n_{2} ; \quad n=d \delta, \quad n_{1}=d_{1} \delta_{1}, \quad n_{2}=d_{2} \delta_{2} ; \\
& 2 n^{\prime}=m_{1}{ }^{\prime}+m_{2}^{\prime} ; \quad n^{\prime}=t^{\prime} \tau^{\prime}, \quad m_{1}^{\prime}=t_{1}^{\prime} \tau_{1}^{\prime}, \quad m_{2}^{\prime}=t_{2}^{\prime} \tau_{2}^{\prime}: \\
& \sum\left[f\left(\left(d_{1}+d_{2}\right)\left(t_{1}^{\prime}-t_{2}^{\prime}\right),\left(\delta_{1}-\delta_{2}\right)\left(\tau_{1}^{\prime}+\tau_{2}^{\prime}\right)\right)\right. \\
& \quad-f\left(\left(d_{1}+d_{2}\right)\left(t_{1}^{\prime}+t_{2}^{\prime}\right),\left(\delta_{1}-\delta_{2}\right)\left(\tau_{1}^{\prime}-\tau_{2}^{\prime}\right)\right) \\
& \quad-f\left(\left(d_{1}-d_{2}\right)\left(t_{1}^{\prime}-t_{2}^{\prime}\right),\left(\delta_{1}+\delta_{2}\right)\left(\tau_{1}^{\prime}-\tau_{2}^{\prime}\right)\right) \\
& \left.\quad+f\left(\left(d_{1}-d_{2}\right)\left(t_{1}^{\prime}+t_{2}^{\prime}\right),\left(\delta_{1}+\delta_{2}\right)\left(\tau_{1}^{\prime}-\tau_{2}^{\prime}\right)\right)\right] \\
& =\sum t^{\prime}\left[2(d-1) f(0,0)-(d+2 \delta-3)\left\{f\left(2 d t^{\prime}, 0\right)+f\left(0,2 d t^{\prime}\right)\right\}\right. \\
& \left.+2 \sum_{r=1}^{\delta-1}\left\{f\left(2 r t^{\prime}, 0\right)+f\left(0,2 r t^{\prime}\right)\right\}\right] .
\end{aligned}
$$

As indicated by the notation, the $t_{1}, \tau_{1}$ occurring in the arguments of $f$ in (I) are conjugate divisors of $m_{1}$, and likewise for $t_{2}, \tau_{2}$, and $m_{2}$. The point to be noticed is that the $t_{1}, \tau_{1}$ occurring in the argument of $f$ are conjugate, and are not taken from different decompositions of $m_{1}$. Similar remarks apply to all the identities.

The arguments in $\left(\mathrm{I}, \mathrm{I}^{\prime}\right)$ are bilinear in the arguments occurring in (I) and $\left(\mathrm{I}^{\prime}\right)$, and similarly for $\left(\mathrm{II}, \mathrm{I}^{\prime}\right)$. We call $\left(\mathrm{I}, \mathrm{I}^{\prime}\right)$ the composition of (I) with itself, or the duplication of (I), and (II, $\left.\mathrm{I}^{\prime}\right)$ the composition of (II) with (I). Note that order is relevant in the symbol (II, $\left.\mathrm{I}^{\prime}\right)$; it will be seen that $\left(\mathrm{I}^{\prime}, \mathrm{II}\right)$ is meaningless. The general problem suggested by these examples can now be 
stated. Given s arithmetical identities concerning parity functions summed over quadratic partitions, to construct from them identities concerning parity functions whose arguments are of degree $s$, are products of the arguments of the functions in the given identities, and are linear in the arguments of the respective identities. The resulting identities will be called compositions of the given identities. This may be extended in obvious ways to the compounding of compositions. It will be sufficient here to indicate the algebraic method by which the above examples were obtained. Although elementary arithmetical proofs can be given, the general algebraic method is preferable on account of its flexibility, suggestiveness in finding compositions, and ease of application.

2. Algebraic Method. The identity (I) is the paraphrase of the following identity* in elliptic theta functions:

$$
\begin{gathered}
\phi_{100}(x, y) \phi_{100}(x,-y)=\psi_{10}^{2}(x)-\psi_{10}^{2}(y), \\
\phi_{100}(x, y) \equiv \vartheta_{1}^{\prime} \vartheta_{1}(x+y) /\left(\vartheta_{0}(x) \vartheta_{0}(y)\right), \\
\psi_{10}(x)=\vartheta_{2} \vartheta_{3} \vartheta_{1}(x) / \vartheta_{0}(x),
\end{gathered}
$$

for which the requisite expansions are

$$
\begin{array}{rlrl}
\phi_{100}(x, y) & =4 \sum_{m} q^{m / 2} \sum \sin (t x+\tau y), & & (m=t \tau), \\
\psi_{10}{ }^{2}(x) & =4 \sum_{n} q^{n}\left[\sigma_{1}(n)-2 \sum t \cos 2 t x\right], \quad(n=t \tau),
\end{array}
$$

the $m, n, t, \tau$ notation being as in $\$ 1$.

The identity (II) is the paraphrase of

$$
\begin{gathered}
\phi_{111}(x, y) \phi_{111}(x,-y)=\psi_{01}^{2}(x)-\psi_{01}{ }^{2}(y), \\
\phi_{111}(x, y) \equiv \vartheta_{1}^{\prime} \vartheta_{1}(x+y) / \vartheta_{1}(x) \vartheta_{1}(y), \psi_{01}(x) \equiv \vartheta_{2} \vartheta_{3} \vartheta_{0}(x) / \vartheta_{1}(x), \\
\phi_{111}(x, y)=\operatorname{ctn} x+\operatorname{ctn} y+4 \sum_{n} q^{2 n} \sum \sin 2(d x+\delta y), \\
\quad(n=d \delta), \\
\psi_{01}^{2}(x)=\csc ^{2} x+4 \sum_{n} q^{n} \sigma_{1}(n)-8 \sum_{n} q^{2 n} \sum d \cos 2 d x, \\
(n=d \delta) .
\end{gathered}
$$

\footnotetext{
* Several examples of the process of paraphrasing are given in the series of notes, Quadratic partitions, appearing in this Bulletin; the last note is in vol. 39 (1933). The series is to be continued.
} 
Obviously

$$
F(x, y) \equiv \phi_{100}(x u, y v) \phi_{100}(x u,-y v),
$$

where $u, v$ are independent variables, is finite and single-valued for all pairs of integer values of $x, y$, and

$$
F(x, y)=F(-x, y)=F(x,-y) .
$$

Hence we may take $f(x, y)=F(x, y)$ in the left of (I) and, by (1),

$$
f(x, y)=\psi_{10}^{2}(x u)-\psi_{10}^{2}(y v)
$$

in the right of (I). Equating coefficients of like powers of $q$ in the resulting identity, we obtain a trigonometric identity in $u, v$ which paraphrases into $\left(\mathrm{I}, \mathrm{I}^{\prime}\right)$. In the same way (II, $\mathrm{I}^{\prime}$ ) follows from (2). Note that (I, $\left.\mathrm{II}^{\prime}\right)$ which, if it existed, would be the result of taking

$$
f(x, y)=\phi_{111}(x u, y v) \phi_{111}(x u,-y v)
$$

in the left of (I), and

$$
f(x, y)=\psi_{01}^{2}(x u)-\psi_{01}^{2}(y v)
$$

in the right, is meaningless, since $\phi_{111}(x, y)$ is undefined if $x y=0$. From these examples the general process is evident. The symbolic algebra for performing the compositions, which is suggested by comparing the left hand members of the given identities with their compositions, will be developed in another paper, where further examples will be given. The partitions in a given set of identities to be compounded may be of any degrees (the degree is the number of independent variables). The number of independent variables may be increased or diminished by composition.

California Institute of Technology 